\title{
CAMINHONEIRO DE TRANSPORTE RODOVIÁRIO DE CARGAS: BUSCA DE TRATAMENTOS ODONTOLÓGICOS
}

\author{
ROAD TRANSPORTATION TRUCK DRIVER: SEARCH OF DENTISTRY \\ TREATMENTS.
}

\section{CAMINADOR DE TRANSPORTE POR CARGO: BÚSQUEDA DE TRATAMIENTOS ODONTOLÓGICOS}

Recebimento: 03/02/2018- Aceite: 09/09/2018- Publicação: 28/10/2018

Processo de Avaliação: Double Blind Review

Nellis Oliveira Santos ${ }^{1}$

Mestre em Políticas Públicas

Universidade Mogi das Cruzes- UMC

nellissantos@umc.br

Luiz Teruo Kawamoto Jr

Doutor em Engenharia Biomédica

Instituto Federal de São Paulo- IFSP

luizteruo@hotmail.com

Hewely Cardoso

Especialização em Estratégia de Marketing e Comunicação

Universidade Mogi das Cruzes- UMC

hewely@umc.br

Claudio José Carvajal Jr.

Doutor em Engenharia Biomédica

Faculdade Informática e Administração Paulista - FIAP

prof.claudiocarvajal@gmail.com

Luci Mendes Bonini

Doutora em Comunicação e Semiotica

Universidade Mogi das Cruzes- UMC

luci.bonini@umc.br

Waltraudi Orchulhak Kawamoto

Doutora em Engenharia Biomédica

Universidade do Brasil

waltraudikawa@gmail.com

\footnotetext{
${ }^{1}$ Autor para correspondência: UMC - Universidade Mogi das Cruzes- Av. Dr. Cândido Xavier de Almeida Souza, 200 - Centro Cívico, Mogi das Cruzes - SP, 08780-911-Brasil
} 


\title{
RESUMO
}

O objetivo desta pesquisa foi explorar hábitos dos caminhoneiros na busca por tratamentos odontológicos. O método da pesquisa utilizado foi quantitativo, exploratório-descritivo e de corte transversal, com 270 sujeitos de pesquisa. O estudo incluiu caminhoneiros com idade de vinte e um a sessenta anos que atuam no ramo de transporte rodoviário de cargas e trafegam na Rodovia Presidente Dutra. Apesar de não estatisticamente diferente, foi detectada tendência de quanto maior o grau de instrução, maior a renda; e quanto maior o grau de instrução, são buscadas melhores formas de tratamentos odontológicos; o fator renda não teve influência forma busca de tratamento odontológico. Como sugestão de futura pesquisa, fazer uma pesquisa mais ampla, de forma a possibilitar melhores análises estatísticas.

Palavras chaves: políticas públicas; saúde bucal; tratamentos odontológicos.

\begin{abstract}
The objective of this research was to explore truckers' habits in the search for dental treatments. The research method used was quantitative, exploratory-descriptive and crosssectional, with 270 subjects. The study included truck drivers from the age of twenty one to sixty who work in the field of road transportation of cargo and travel on the Presidente Dutra Highway. Although not statistically different, a trend was detected that the higher the education level, the higher the income; and the higher the level of education, the better forms of dental treatment are sought; the income factor had no influence form seeking dental treatment. As a suggestion of future research, make a broader survey, in order to enable better statistical analysis.
\end{abstract}

Key words: public policies; oral health; dental treatments.

\section{RESUMEN:}

El objetivo de esta investigación fue explorar hábitos de los camioneros en la búsqueda de tratamientos odontológicos. El método de la investigación utilizada fue cuantitativo, exploratorio-descriptivo y de corte transversal, con 270 sujetos de investigación. El estudio incluyó camioneros con edad de veintiún a sesenta años que actúan en el ramo de transporte por carretera de cargas y viajan en la Ruta Presidente Dutra. A pesar de no estadísticamente diferente, fue detectada tendencia de cuanto mayor el grado de instrucción, mayor la renta; y cuanto mayor el grado de instrucción, se buscan mejores formas de tratamientos odontológicos; el factor renta no tuvo influencia forma búsqueda de tratamiento odontológico. Como sugerencia de futura investigación, hacer una investigación más amplia, para posibilitar mejores análisis estadísticos.

Palabras claves: políticas públicas; salud bucal; tratamientos odontológicos. 


\section{INTRODUÇÃO}

Penteado et al. (2008), em seu estudo sobre as condicionantes laborais dos caminhoneiros afirma que estes agem de forma negativa em relação a sua qualidade de vida. “A informalidade e a precarização presentes nessa profissão, configuradas pela terceirização, comprometem as condições de trabalho e saúde dos trabalhadores submetidos ao mesmo contexto." (SILVA et al. 2016 p. 153).

Vendrame (2016), afirma que a relação entre qualidade de vida e saúde bucal é indissociável. Sobrinho (2014) ratifica que a saúde inicia-se pela boca, daí a importância que deve ser dada na prevenção e tratamento odontológico.

Nas Diretrizes da Política Nacional de Saúde Bucal de 2004, descreve que é preciso conhecer as particularidades do perfil epidemiológico da população bem como suas condições socioeconômicas e hábitos de vida para um melhor atendimento de suas necessidades de saúde, especialmente os trabalhadores que possuem dificuldades de acesso aos postos de saúde e principalmente dentro dos horários de atendimento desses serviços (BRASIL, 2004).

Mota et. al. (2015), afirmam que no Brasil, com toda busca empenhada na regulamentação para Políticas Públicas direcionadas para saúde bucal do trabalhador, não possibilitou a introdução do cirurgião-dentista na atuação de uma equipe de saúde no Programa de Controle Médico de Saúde Ocupacional (PCMSO).

Segundo Vieira et. al. (2013), são raros os estudos que se ocuparam da percepção que os trabalhadores do setor têm sobre esse processo, notadamente na área de saúde bucal.

O objetivo geral desta pesquisa foi explorar hábitos dos caminhoneiros na busca por tratamentos odontológicos.

\section{REFERENCIAL TEÓRICO}

No estudo realizado por Lima, L. et al. (2015), região metropolitana de Salvador, Bahia, por meio do Programa de Saúde Bucal na Empresa (PSBE) entre 2003 a 2008 e Serviço Social da Indústria (SESI) da Bahia, sobre as condições de saúde bucal dos trabalhadores em cinco indústrias, onde o programa apresentava uma proposta de atenção à saúde bucal, foi observado que houve acesso dos trabalhadores ao atendimento odontológico em consultórios instalados dentro das referentes empresas estudadas, buscando trazer aos trabalhadores a importância sobre mudanças de hábitos e comportamentos referente a saúde bucal.

De acordo Sobrinho (2014, p. 203) os dados CPO-D (dentes cariados, perdidos e obturados) apresentados pelo relatório SBBrasil 2010 "permitem afirmar que houve 
ampliação do acesso da população adulta ( 35 a 44 anos) ao tratamento da cárie dentária, pois em 2003 a média de dentes obturados era de 4,22, passando a 7,33 em 2010”.

Por meio do estudo de Guedes et al. (2012, p. 349), foi observado entre os caminhoneiros que 23,8\% possuíam ensino fundamental completo, 19,3\% ensino médio completo e 1,6\% ensino superior completo. "[...] levou os autores a sugerir que o nível de escolaridade pode impactar diretamente sobre os hábitos de saúde do indivíduo".

Como observado sobre a saúde bucal de acordo estudo de Guedes et al. (2012), 8,7\% dos caminhoneiros conhecem o serviço para essa área da saúde e apenas 5,5\% utilizam esse serviço. No mesmo estudo foram apresentados os seguintes resultados sobre as escolhas para atendimento referente à saúde e saúde bucal.

Sampaio e Cruz (2015) em um estudo sobre a população adulta brasileira com idade entre 35 a 44 anos, e amostra de 8.855 sujeitos por meio do banco de dados com abrangência nacional do Projeto Saúde Bucal Brasil 2010, observaram que os usuários de serviços odontológicos público ou privado possuíam renda familiar entre $\mathrm{R} \$ 501,00$ a $\mathrm{R} \$ 1.500,00$ e escolaridade de 9 a 12 anos de estudo.

Lima e Oliveira (2017), conforme estudo realizado com o objetivo em avaliar a auto percepção em saúde bucal em caminhoneiros homens, identificaram que $83,7 \%$ da renda familiar dos caminhoneiros era de $\mathrm{R} \$ 2.501,00$ e $\mathrm{R} \$ 4.500,00$. Observa-se também que os caminhoneiros que consultaram o dentista pela última vez: $62,5 \%$ com ensino fundamental incompleto foram no particular, 59,6\% com ensino fundamental completo foram no dentista particular, $63,4 \%$ com ensino médio foram no dentista particular e $71,4 \%$ foram no dentista do serviço público. Nota-se que o motivo da última consulta houve um crescimento considerável no item revisão/prevenção de uma categoria de ensino para outra, iniciando em $43,8 \%$ com ensino fundamental incompleto e evoluindo à $85,7 \%$ no ensino superior (LIMA e OLIVEIRA, 2017).

Conforme a renda do indivíduo aumenta, ele passa e ser conveniado de planos de saúde no sistema privado e, quanto menor a renda, aliado ao menor grau de escolaridade, maior será a procura da população por serviços públicos e serviços odontológicos do SUS (CARDOZO et al. 2016).

No estudo de Sampaio e Cruz (2014 p.21), "Foi observado que o serviço odontológico privado foi o mais utilizado pela população adulta brasileira em todas as regiões geográficas", sendo que $59,6 \%$ buscou o serviço odontológico privado e 40,4\% buscou o serviço odontológico público. 
Segundo Chaves e Santos (2017), a Política Nacional de Saúde Bucal proporcionou um aumento na provisão e cobertura de serviços odontológicos públicos entre 2003 e 2006 e que as taxas foram mantidas em períodos subsequentes (2007 a 2010 e 2011 a 2014). Houve uma expansão no financiamento do governo, recursos humanos e infraestrutura. O montante de recursos transferidos para os governos estaduais e municipais aumentou de 83,4 milhões em 2003 para 916 milhões em 2014, o equivalente a um aumento de 10,9 vezes. No entanto, o uso de serviços odontológicos públicos permaneceu estável, com um leve aumento de 29,7\% em 2003 para 30,7\% em 2008, enquanto a utilização de serviços privados aumentou de 64,4\% em 2003 para 74,3\% em 2013.

Para Miotto, Silotti e Barcellos (2012), em estudo feito aleatoriamente com 170 sujeitos na Prefeitura Municipal na cidade de Venda Nova do Imigrante (ES), com o objetivo de analisar se a prevalência da dor dentária gerava o absenteísmo, encontraram-se possíveis associações com características sociodemográficas. Foi identificado com esse estudo que, “A prevalência de dor encontrada demonstra a necessidade de políticas de promoção de saúde bucal para melhorar a qualidade de vida dos trabalhadores".

De acordo com Guerra e Leite (2014), em um estudo transversal com 326 trabalhadores que responderam as questões do OHIP-14 sobre autoavaliação de saúde, morbidade bucal e questões socioeconômicas e demográficas. A análise de regressão linear mostrou que cerca de $40 \%$ do impacto da saúde bucal na qualidade de vida pode ser explicado pelas variáveis: escolaridade $(\mathrm{p}=0,03)$, idade $(\mathrm{p}=0,03)$, motivo da visita ao dentista $(\mathrm{p}=$ 0,01 percepção de saúde bucal ( $p<0,01)$ e satisfação com dentes e boca ( $p<0,01)$.

Pesquisa de Lima et. al. (2015) com objetivo de verificar as condições de saúde bucal de trabalhadores de cinco indústrias da região metropolitana de Salvador, Bahia, que participavam do Programa de Saúde Bucal na Empresa (PSBE) entre os anos 2003 a 2008, mostrou que a redução na incidência de cáries e na necessidade de próteses entre os que mais realizaram exames indica que programas de saúde bucal nas empresas podem contribuir para a melhor saúde bucal dos trabalhadores.

\section{MÉTODO}

Por meio das buscas sobre estudos realizados de forma aprofundada para algumas vertentes apresentando determinadas prevalências referentes a saúde e hábitos dos caminhoneiros, o método da pesquisa utilizado foi quantitativo, exploratório-descritivo e de corte transversal. Aplicou-se à um questionário sócio demográfico. 
Primeiro foi feita revisão bibliográfica descritiva. As buscas sobre as referências bibliográficas foram investigadas por meio de bases de pesquisas, sendo DEDALUS, Scielo, SibiUSP, Portal Regional da BVS, BIREME, LILACS, Portal Brasil e periódicos CAPES.

O estudo incluiu caminhoneiros com idade de vinte e um (21) anos a sessenta e cinco (65) anos que atuam no ramo de transporte rodoviário de cargas e utilizam a Rodovia Presidente Dutra - BR 116, que liga o Estado de São Paulo ao Estado do Rio de Janeiro.

Foram considerados os sujeitos com idade mínima de vinte um (21) anos em consonância aos pré-requisitos do CONTRAN - Conselho Nacional de Trânsito na resolução $\mathrm{n}^{\circ} 168$, de 14 de dezembro de 2004, item 6.3.2, e lei $\mathrm{n}^{\circ} 9.503$ de setembro de 1997, para exigência na aquisição da $\mathrm{CNH}$ - carteira nacional de habilitação, categoria "E".

Foi levado em consideração para esse estudo, a exclusão dos sujeitos com dezoito (18) anos a vinte (20) anos e onze (11) meses de acordo com os pré-requisitos do CONTRAN e lei $\mathrm{n}^{\circ} 9.503$, não poderiam estar pilotando combinação de veículos em que a unidade tratora se combine na categoria " $\mathrm{D}$ " e unidade articulada tenha $6.000 \mathrm{~kg}$ (seis mil quilogramas) ou mais de peso bruto total.

A logística para coleta de dados ocorreu no trecho da Rodovia Presidente Dutra. Os pontos de coletas ocorreram de forma aleatória em postos de combustíveis que tinham caminhões estacionados, entre as cidades de Arujá e Aparecida.

O projeto passou pelo Comitê de Ética em Pesquisa, número do parecer: 2.292.320. Conforme o parecer do projeto, todos os termos estão de acordo com as Resoluções de pesquisa com seres humanos, os objetivos estão claros e o método proposto é suficiente para atingir os objetivos propostos.

Em posse das fichas de respostas foi utilizado o programa BioEstat 5.0 para tabular dados. De forma descritiva foram apresentados dados na relação entre a profissão do caminhoneiro e a busca por tratamento dentário.

\section{RESULTADOS}

Dos caminhoneiros profissionais de cargas rodoviárias pesquisados, 270 sujeitos consentiram em participar desta pesquisa (100\%).

Para tabular os dados utilizou-se o programa BioEstat 5.0. Foi realizado o teste de Qui-Quadrado. Nível de decisão: alfa = 0,01. 
Um sujeito se recusou a responder seu grau de instrução $(n=1 / 0,37 \%)$, logo nos resultados onde envolva grau de instrução a amostra em consideração é de 269 sujeitos e nos outros itens tabulados a amostra foi total, 270 sujeitos.

A Tabela 1 exibe os resultados absolutos, e a Tabela 2 os resultados em percentuais, coletados na pesquisa de campo e distribuídos por frequência na faixas etárias e grau de instrução.

Tabela 1 - Distribuição dos valores absolutos: quantidade de sujeitos por faixa etária e grau de instrução.

\begin{tabular}{|c|c|c|c|c|c|c|}
\hline \multicolumn{7}{|c|}{ ATÉ ENSINO FUNDAMENTAL } \\
\hline $21-29$ & 30-39 & $40-49$ & $50-59$ & $60-69$ & 70-79 & TOTAL \\
\hline 2 & 22 & 49 & 45 & 14 & 1 & 133 \\
\hline \multicolumn{7}{|c|}{ ATÉ O ENSINO MÉDIO } \\
\hline 21-29 & 30-39 & $40-49$ & $50-59$ & $60-69$ & $70-79$ & TOTAL \\
\hline 16 & 42 & 39 & 19 & 1 & 6 & 123 \\
\hline \multicolumn{7}{|c|}{ ATÉ O ENSINO SUPERIOR } \\
\hline 21-29 & $30-39$ & $40-49$ & $50-59$ & $60-69$ & 70-79 & TOTAL \\
\hline 0 & 3 & 4 & 6 & 0 & 0 & 13 \\
\hline
\end{tabular}

Fonte: elaborado pelos autores (2018).

Tabela 2 - Porcentagens: por faixa etária e grau de instrução.

\begin{tabular}{|c|c|c|c|}
\hline IDADE & $\begin{array}{c}\text { ATÉ O ENSINO } \\
\text { FUNDAMENTAL }\end{array}$ & $\begin{array}{c}\text { ATÉ O ENSINO } \\
\text { MÉDIO }\end{array}$ & $\begin{array}{c}\text { ATÉ O ENSINO } \\
\text { SUPERIOR }\end{array}$ \\
\hline $\mathbf{2 1 - 2 9}$ & $1.50 \%$ & $13.01 \%$ & $0.00 \%$ \\
\hline $\mathbf{3 0 - 3 9}$ & $16.54 \%$ & $34.15 \%$ & $23.08 \%$ \\
\hline $\mathbf{4 0 - 4 9}$ & $36.84 \%$ & $31.71 \%$ & $30.77 \%$ \\
\hline $\mathbf{5 0 - 5 9}$ & $33.83 \%$ & $15.45 \%$ & $46.15 \%$ \\
\hline $\mathbf{6 0 - 6 9}$ & $10.53 \%$ & $0.81 \%$ & $0.00 \%$ \\
\hline $\mathbf{7 0 - 7 9}$ & $0.75 \%$ & $4.88 \%$ & $0.00 \%$ \\
\hline
\end{tabular}

Fonte: elaborado pelos autores (2018). 
A Tabela 3 apresenta os resultados absolutos, a Tabela 4 em percentuais, e a Figura 1 a análise estatística, coletados na pesquisa de campo e distribuídos por frequência quanto ao grau de instrução, faixa etária e renda.

Tabela 3 - Distribuição dos valores absolutos: grau de instrução e renda.

\begin{tabular}{|c|c|c|c|c|c|c|c|}
\hline \multicolumn{8}{|c|}{ Grau de Instrução versus renda } \\
\hline Alternativa do questionário & $\begin{array}{l}R \$ 0,00- \\
1.000,00\end{array}$ & $\begin{array}{c}\text { R\$ } 1.000,01 \text { - } \\
2.000,00\end{array}$ & $\begin{array}{c}\mathrm{R} \$ 2.000,01 \text { - } \\
3.000,00\end{array}$ & $\begin{array}{c}\text { R\$ } 3.000,01 \text { - } \\
4.000,00\end{array}$ & $\begin{array}{c}R \$ 4.000,01 \\
-8.000,00\end{array}$ & $\begin{array}{c}\text { Acima de } \mathrm{R} \$ \\
8.000,00\end{array}$ & $\begin{array}{c}\text { Total de } \\
\text { respondentes }\end{array}$ \\
\hline Ensino Fundamental completo & 2 & 27 & 45 & 34 & 20 & 5 & 133 \\
\hline Ensino Médio completo & 1 & 14 & 41 & 35 & 18 & 14 & 123 \\
\hline Ensino Superior completo & 0 & 1 & 3 & 4 & 1 & 4 & 13 \\
\hline
\end{tabular}

Fonte: elaborado pelos autores (2018).

Tabela 4 - Porcentagens: grau de instrução e renda.

\begin{tabular}{|c|c|c|c|c|c|c|c|}
\hline \multicolumn{8}{|c|}{ Grau de Instrução versus renda } \\
\hline Alternativa do questionário & $\begin{array}{l}R \$ 0,00 \text { - } \\
1.000,00\end{array}$ & $\begin{array}{c}\text { RS } 1.000,01 \text { - } \\
2.000,00\end{array}$ & $\begin{array}{c}\mathrm{R} \$ 2.000,01 \text { - } \\
3.000,00\end{array}$ & $\begin{array}{c}\text { R\$ } 3.000,01 \text { - } \\
4.000,00\end{array}$ & $\begin{array}{c}\text { R\$ } 4.000,01 \\
-8.000,00\end{array}$ & \begin{tabular}{|c|} 
Acima de $\mathbf{R} \$$ \\
$8.000,00$
\end{tabular} & $\begin{array}{c}\text { Total de } \\
\text { respondentes }\end{array}$ \\
\hline Ensino Fundamental completo & $1.50 \%$ & $20.30 \%$ & $33.83 \%$ & $25.56 \%$ & $15.04 \%$ & $3.76 \%$ & $100.00 \%$ \\
\hline Ensino Médio completo & $0.81 \%$ & $11.38 \%$ & $33.33 \%$ & $28.46 \%$ & $14.63 \%$ & $11.38 \%$ & $100.00 \%$ \\
\hline Ensino Superior completo & $0.00 \%$ & $7.69 \%$ & $23.08 \%$ & $30.77 \%$ & $7.69 \%$ & $30.77 \%$ & $100.00 \%$ \\
\hline
\end{tabular}

Fonte: elaborado pelos autores (2018).

Figura 1 - Análise estatística dos valores absolutos: grau de instrução e renda.

\begin{tabular}{l} 
[Nan Teste do Qui-Quadrado: Partição \\
Arquivo Editar Gráfico \\
\hline
\end{tabular}

Fonte: elaborado pelos autores (2018).

A Tabela 5 apresenta os resultados absolutos, e a Tabela 6 em percentuais, coletados na pesquisa de campo e distribuídos por frequência quanto ao grau de instrução, faixa etária e renda. 
Tabela 5 - Distribuição dos valores absolutos: grau de instrução, faixa etária e renda.

\begin{tabular}{|c|c|c|c|c|c|c|}
\hline Ensino/faixa etária & $\begin{array}{l}R \$ 0,00- \\
1.000,00\end{array}$ & $\begin{array}{c}R \$ \\
1.000,01- \\
2.000,00 \\
\end{array}$ & $\begin{array}{c}R \$ \\
2.000,01- \\
3.000,00 \\
\end{array}$ & $\begin{array}{c}R \$ \\
3.000,01- \\
4.000,00 \\
\end{array}$ & $\begin{array}{c}R \$ \\
4.000,01- \\
8.000,00 \\
\end{array}$ & $\begin{array}{c}\text { Acima de } \\
R \$ \\
8.000,00\end{array}$ \\
\hline Até o ensino fundamental de 21 a 29 anos & 0 & 0 & 2 & 0 & 0 & 0 \\
\hline Até o ensino Médio de 21 a 29 anos & 0 & 2 & 6 & 5 & 3 & 0 \\
\hline Até o ensino Superior de 21 a 29 anos & 0 & 0 & 0 & 0 & 0 & 0 \\
\hline Até o ensino fundamental de 30 a 39 anos & 1 & 5 & 7 & 6 & 2 & 1 \\
\hline Até o ensino Médio de 30 a 39 anos & 0 & 6 & 13 & 12 & 8 & 4 \\
\hline Até o ensino Superior de 30 a 39 anos & 0 & 0 & 1 & 1 & 0 & 0 \\
\hline Até o ensino fundamental de 40 a 49 anos & 0 & 10 & 18 & 10 & 8 & 1 \\
\hline Até o ensino Médio de 40 a 49 anos & 1 & 2 & 14 & 10 & 3 & 5 \\
\hline Até o ensino Superior de 40 a 49 anos & 0 & 0 & 1 & 2 & 0 & 2 \\
\hline Até o ensino fundamental de 50 a 59 anos & 0 & 10 & 14 & 17 & 5 & 3 \\
\hline Até o ensino Médio de 50 a 59 anos & 0 & 2 & 4 & 7 & 3 & 4 \\
\hline Até o ensino Superior de 50 a 59 anos & 0 & 1 & 0 & 2 & 1 & 2 \\
\hline Até o ensino fundamental de 60 a 65 anos & 0 & 2 & 4 & 0 & 1 & 1 \\
\hline Até o ensino Médio 60 a 65 anos & 1 & 2 & 3 & 2 & 4 & 2 \\
\hline Até o ensino Superior 60 a 65 anos & 0 & 0 & 0 & 0 & 0 & 0 \\
\hline
\end{tabular}

Fonte: elaborado pelos autores (2018).

Tabela 6 - Porcentagens: grau de instrução, faixa etária e renda.

\begin{tabular}{|c|c|c|c|c|c|c|}
\hline \multirow{3}{*}{ Ensino/faixa etária } & \multirow{3}{*}{$\begin{array}{l}R \$ 0,00- \\
1.000,00\end{array}$} & $\mathbf{R} \$$ & $\mathbf{R} \$$ & $\mathbf{R} \$$ & $\mathbf{R} \$$ & \multirow{3}{*}{$\begin{array}{c}\text { Acima de } \\
\mathbf{R} \$ \\
8.000,00\end{array}$} \\
\hline & & $1.000,01-$ & $2.000,01-$ & $3.000,01-$ & $4.000,01$ & \\
\hline & & $2.000,00$ & $3.000,00$ & $4.000,00$ & $8.000,00$ & \\
\hline Até o ensino fundamental de 21 a 29 anos & $0.00 \%$ & $0.00 \%$ & $0.74 \%$ & $0.00 \%$ & $0.00 \%$ & $0.00 \%$ \\
\hline Até o ensino Médio de 21 a 29 anos & $0.00 \%$ & $0.74 \%$ & $2.23 \%$ & $1.86 \%$ & $1.12 \%$ & $0.00 \%$ \\
\hline Até o ensino Superior de 21 a 29 anos & $0.00 \%$ & $0.00 \%$ & $0.00 \%$ & $0.00 \%$ & $0.00 \%$ & $0.00 \%$ \\
\hline Até o ensino fundamental de 30 a 39 anos & $0.37 \%$ & $1.86 \%$ & $2.60 \%$ & $2.23 \%$ & $0.74 \%$ & $0.37 \%$ \\
\hline Até o ensino Médio de 30 a 39 anos & $0.00 \%$ & $2.23 \%$ & $4.83 \%$ & $4.46 \%$ & $2.97 \%$ & $1.49 \%$ \\
\hline Até o ensino Superior de 30 a 39 anos & $0.00 \%$ & $0.00 \%$ & $0.37 \%$ & $0.37 \%$ & $0.00 \%$ & $0.00 \%$ \\
\hline Até o ensino fundamental de 40 a 49 anos & $0.00 \%$ & $3.72 \%$ & $6.69 \%$ & $3.72 \%$ & $2.97 \%$ & $0.37 \%$ \\
\hline Até o ensino Médio de 40 a 49 anos & $0.37 \%$ & $0.74 \%$ & $5.20 \%$ & $3.72 \%$ & $1.12 \%$ & $1.86 \%$ \\
\hline Até o ensino Superior de 40 a 49 anos & $0.00 \%$ & $0.00 \%$ & $0.37 \%$ & $0.74 \%$ & $0.00 \%$ & $0.74 \%$ \\
\hline Até o ensino fundamental de 50 a 59 anos & $0.00 \%$ & $3.72 \%$ & $5.20 \%$ & $6.32 \%$ & $1.86 \%$ & $1.12 \%$ \\
\hline Até o ensino Médio de 50 a 59 anos & $0.00 \%$ & $0.74 \%$ & $1.49 \%$ & $2.60 \%$ & $1.12 \%$ & $1.49 \%$ \\
\hline Até o ensino Superior de 50 a 59 anos & $0.00 \%$ & $0.37 \%$ & $0.00 \%$ & $0.74 \%$ & $0.37 \%$ & $0.74 \%$ \\
\hline Até o ensino fundamental de 60 a 65 anos & $0.00 \%$ & $0.74 \%$ & $1.49 \%$ & $0.00 \%$ & $0.37 \%$ & $0.37 \%$ \\
\hline Até o ensino Médio 60 a 65 anos & $0.37 \%$ & $0.74 \%$ & $1.12 \%$ & $0.74 \%$ & $1.49 \%$ & $0.74 \%$ \\
\hline Até o ensino Superior 60 a 65 anos & $0.00 \%$ & $0.00 \%$ & $0.00 \%$ & $0.00 \%$ & $0.00 \%$ & $0.00 \%$ \\
\hline
\end{tabular}

Fonte: elaborado pelos autores (2018).

A Tabela 7 apresenta os resultados absolutos, a Tabela 8 em percentuais, e a Figura 2 a análise estatística, coletados na pesquisa de campo e distribuídos por frequência, grau de instrução e onde os caminhoneiros realizam seus tratamentos dentários. 
Tabela 7 - Distribuição dos valores absolutos: Grau de instrução e tratamentos dentário.

\begin{tabular}{|l|c|c|c|c|c|c|}
\hline \multicolumn{7}{|c|}{ Grau de Instrução e onde realiza tratamento dentário } \\
\hline Alternativa do questionário & $\begin{array}{c}\text { Dentista } \\
\text { Particular }\end{array}$ & $\begin{array}{c}\text { Denstista do } \\
\text { Convênio }\end{array}$ & $\begin{array}{c}\text { Dentista do posto } \\
\text { de saúde }\end{array}$ & $\begin{array}{c}\text { Retornando em } \\
\text { campanhas } \\
\text { existentes nas } \\
\text { estradas }\end{array}$ & Outros & $\begin{array}{c}\text { Total de } \\
\text { respondentes }\end{array}$ \\
\hline Ensino Fundamental completo & 77 & 19 & 25 & 2 & 10 & 133 \\
\hline Ensino Médio completo & 88 & 19 & 11 & 2 & 3 & 123 \\
\hline Ensino Superior completo & 6 & 3 & 2 & 1 & 1 & 13 \\
\hline
\end{tabular}

*Outros: Citado pelos caminhoneiros - SEST SENAT e ponto de tratamento dentário gratuito na concessionária da rodovia.

Fonte: elaborado pelos autores (2018). 
Tabela 8 - Porcentagens: Grau de instrução e tratamentos dentários.

\begin{tabular}{|l|c|c|c|c|c|c|}
\hline \multicolumn{7}{|c|}{ Grau de Instrução e onde realiza tratamento dentário } \\
\hline Alternativa do questionário & $\begin{array}{c}\text { Dentista } \\
\text { Particular }\end{array}$ & $\begin{array}{c}\text { Denstista do } \\
\text { Convênio }\end{array}$ & $\begin{array}{c}\text { Dentista do posto } \\
\text { de saúde }\end{array}$ & $\begin{array}{c}\text { Retornando em } \\
\text { campanhas } \\
\text { existentes nas } \\
\text { estradas }\end{array}$ & Outros & $\begin{array}{c}\text { Total de } \\
\text { respondentes }\end{array}$ \\
\hline Ensino Fundamental completo & $28.62 \%$ & $7.06 \%$ & $9.29 \%$ & $0.74 \%$ & $3.72 \%$ & $49.44 \%$ \\
\hline Ensino Médio completo & $32.71 \%$ & $7.06 \%$ & $4.09 \%$ & $0.74 \%$ & $1.12 \%$ & $45.72 \%$ \\
\hline Ensino Superior completo & $2.23 \%$ & $1.12 \%$ & $0.74 \%$ & $0.37 \%$ & $0.37 \%$ & $4.83 \%$ \\
\hline
\end{tabular}

*Outros: Citado pelos caminhoneiros - SEST SENAT e ponto de tratamento dentário gratuito na concessionária da rodovia.

Fonte: elaborado pelos autores (2018).

Figura 2 - Análise estatística da distribuição dos valores absolutos: Grau de instrução e tratamentos dentário.

\begin{tabular}{l} 
[L] Teste do Qui-Quadrado: Partição \\
Arquivo Editar Gráfico \\
\hline
\end{tabular}

Fonte: elaborado pelos autores (2018).

A Tabela 9 apresenta os resultados absolutos, a Tabela 10 em percentuais, e a Figura 3 a análise estatística, coletados na pesquisa de campo e distribuídos por frequência, referente a renda dos caminhoneiros e onde realizam os tratamentos dentários. A maior amostra de caminhoneiros que possuem renda entre $\mathrm{R} \$ 2.000,01$ - 3.000,00, está sendo representado por 87 sujeitos. 
Tabela 9 - Distribuição dos valores absolutos: renda do caminhoneiro e onde realizam os tratamentos dentários.

\begin{tabular}{|l|c|c|c|c|c|}
\hline \multicolumn{2}{|c|}{ Renda versus onde realiza tratamento dentário } \\
\hline $\begin{array}{c}\text { Alternativa do } \\
\text { questionário }\end{array}$ & $\begin{array}{c}\text { Dentista } \\
\text { Particular }\end{array}$ & $\begin{array}{c}\text { Denstista do } \\
\text { Convênio } \\
\text { posto de saúde }\end{array}$ & $\begin{array}{c}\text { Retornando em } \\
\text { campanhas } \\
\text { existentes nas } \\
\text { estradas }\end{array}$ & Outros \\
Tespondentes \\
R\$ 0,00-1.000,00
\end{tabular}

*Outros: Citado pelos caminhoneiros - SEST SENAT e ponto de tratamento dentário gratuito na concessionária da rodovia.

Fonte: elaborado pelos autores (2018).

Tabela 10 - Porcentagens: renda do caminhoneiro e onde realizam os tratamentos dentários.

\begin{tabular}{|l|c|c|c|c|c|c|}
\hline \multicolumn{7}{|c|}{ Renda versus onde realiza tratamento dentário } \\
\hline $\begin{array}{c}\text { Alternativa do } \\
\text { questionário }\end{array}$ & $\begin{array}{c}\text { Dentista } \\
\text { Particular }\end{array}$ & $\begin{array}{c}\text { Denstista do } \\
\text { Convênio }\end{array}$ & $\begin{array}{c}\text { Dentista do } \\
\text { posto de saúde }\end{array}$ & $\begin{array}{c}\text { Retornando em } \\
\text { campanhas } \\
\text { existentes nas } \\
\text { estradas }\end{array}$ & Outros & $\begin{array}{c}\text { Total de } \\
\text { respondentes }\end{array}$ \\
\hline R\$ 0,00-1.000,00 & 2 & 0 & 0 & 0 & 1 & 3 \\
\hline R\$ 1.000,01 - 2.000,00 & 24 & 7 & 6 & 2 & 5 & 44 \\
\hline R\$ 2.000,01 - 3.000,00 & 56 & 16 & 13 & 1 & 1 & 87 \\
\hline R\$ 3.000,01 - 4.000,00 & 46 & 10 & 15 & 1 & 2 & 74 \\
\hline R\$ 4.000,01 - 8.000,00 & 29 & 7 & 1 & 0 & 2 & 39 \\
\hline Acima de R\$ 8.000,00 & 15 & 1 & 4 & 1 & 2 & 23 \\
\hline
\end{tabular}

*Outros: Citado pelos caminhoneiros - SEST SENAT e ponto de tratamento dentário gratuito na concessionária da rodovia.

Fonte: elaborado pelos autores (2018). 
Figura 3 - Análise da distribuição dos valores absolutos: renda do caminhoneiro e onde realizam os tratamentos dentários.

\begin{tabular}{|c|c|c|c|c|c|}
\hline \multicolumn{3}{|c|}{ [1] Teste do Qui-Quadrado: Partição } & \multirow[t]{2}{*}{-} & \multirow[t]{2}{*}{$\square$} & \multirow[t]{2}{*}{ X } \\
\hline Arquivo Editar $\mathrm{C}$ & Gráfico & & & & \\
\hline & Lin : Col & $\begin{array}{c}\text { Qui- } \\
\text { Quadrado }\end{array}$ & GL & (p) & \\
\hline Partição 1 & $2: 2$ & 0.5278 & 1 & 0.4675 & \\
\hline Partição 2 & $3: 2$ & 0.0291 & 1 & 0.8644 & \\
\hline Partição 3 & $4: 2$ & 0.3819 & 1 & 0.5366 & \\
\hline Partição 4 & $5: 2$ & 0.0165 & 1 & 0.8977 & \\
\hline Partição 5 & $6: 2$ & 1.6766 & 1 & 0.1954 & \\
\hline Partição 6 & $2: 3$ & 0.2753 & 1 & 0.5998 & \\
\hline Partição 7 & $3: 3$ & 0.0003 & 1 & 0.9871 & \\
\hline Partição 8 & $4: 3$ & 1.2087 & 1 & 0.2716 & \\
\hline Partição 9 & $5: 3$ & 5.2390 & 1 & 0.0221 & \\
\hline Partição 10 & $6: 3$ & 0.3187 & 1 & 0.5724 & \\
\hline Partição 11 & $2: 4$ & 0.2623 & 1 & 0.6086 & \\
\hline Partição 12 & $3: 4$ & 1.8656 & 1 & 0.1720 & \\
\hline Partição 13 & $4: 4$ & 0.2303 & 1 & 0.6313 & \\
\hline Partição 14 & $5: 4$ & 0.6581 & 1 & 0.4172 & \\
\hline Partição 15 & $6: 4$ & 0.9156 & 1 & 0.3386 & \\
\hline Partição 16 & $2: 5$ & 2.9578 & 1 & 0.0855 & \\
\hline Partição 17 & $3: 5$ & 8.9850 & 1 & 0.0027 & \\
\hline Partição 18 & $4: 5$ & 0.6612 & 1 & 0.4161 & \\
\hline Partição 19 & $5: 5$ & 0.0460 & 1 & 0.8302 & \\
\hline Partição 20 & $6: 5$ & 0.8262 & 1 & 0.3634 & \\
\hline Partição 21 & $2: 6$ & 0.0000 & 1 & 1.0000 & \\
\hline Partição 22 & $3: 6$ & 0.0000 & 1 & 1.0000 & \\
\hline Partição 23 & $4: 6$ & 0.0000 & 1 & 1.0000 & \\
\hline Partição 24 & $5: 6$ & 0.0000 & 1 & 1.0000 & \\
\hline Partição 25 & $6: 6$ & 0.0000 & 1 & 1.0000 & \\
\hline Geral & Tabela & 27.0819 & 25 & 0.3518 & \\
\hline
\end{tabular}

Fonte: elaborado pelos autores (2018).

\section{ANÁLISE E DISCUSSÃO}

Em relação aos caminhoneiros que buscam tratamento dentário, observam-se os seguintes resultados: caminhoneiros com grau de instrução até o ensino médio, $(71,54 \%)$ e aqueles que possuem renda entre $\mathrm{R} \$ 4.000,01$ - $\mathrm{R} \$ 8.000,00$, $(74,36 \%)$ buscam por tratamentos odontológicos em dentista particular; os que possuem até o ensino superior $(23,08 \%)$ e rendimentos entre $\mathrm{R} \$ 2.000,01-\mathrm{R} \$ 3.000,00,(18,39 \%)$ prevalece à procura pelo atendimento no dentista do convênio; a procura pelo dentista do posto de saúde prevalece pelos que possuem até o ensino fundamental $(18,80 \%)$ e os que apresentam renda entre R\$ 3.000,01 - R \$ 4.000,00, (20,27\%); os caminhoneiros com renda entre $\mathrm{R} \$ 1.000,01-\mathrm{R} \$$ $2.000,00,(4,55 \%)$ e aqueles com ensino superior $(7,69 \%)$ prevalece o retorno em campanhas existentes nas estradas ou outros. Guedes et al. (2012, p. 349) sugeriram [...] que o nível de escolaridade pode impactar diretamente sobre os hábitos de saúde do indivíduo". Por meio do 
IBGE, foi observado que, quando elevado o nível de escolaridade, mais alto o volume de consulta ao dentista, (IBGE, 2013).

No Quadro 1 observam se os estudos realizados conforme cada pesquisa em relação às escolhas para os tratamentos odontológicos, Guedes et al. (2012), Próprio Autor, (2017) e Lima e Oliveira (2017) por meio de pesquisa com caminhoneiros, Miotto, Silotti e Barcellos (2012) com funcionários da Prefeitura Municipal na cidade de Venda Nova do Imigrante (ES), Sampaio e Cruz (2015) e Cardozo et al. (2016) com dados dos SB Brasil 2010.

Quadro 1 - Comparações entre estudos para escolha do tratamento odontológico.

\begin{tabular}{|c|c|c|c|c|}
\hline AUTORES & $\begin{array}{c}\text { ATENDIMENTO } \\
\text { PÚBLICO }\end{array}$ & PARTICULAR & CONVÊNIO & OUTROS \\
\hline $\begin{array}{l}\text { Próprio autor, } \\
\text { (2017) }\end{array}$ & $\begin{array}{c}\text { 18,80\%, até o } \\
\text { Ensino } \\
\text { fundamental }\end{array}$ & $\begin{array}{l}71,54 \%, \text { até o } \\
\text { Ensino médio }\end{array}$ & $\begin{array}{l}23,08 \%, \text { até o } \\
\text { Ensino superior }\end{array}$ & $\begin{array}{c}\text { 7,69, até o Ensino } \\
\text { superior }\end{array}$ \\
\hline $\begin{array}{l}\text { Lima e Oliveira } \\
(2017)\end{array}$ & $\begin{array}{l}71,4 \% \text { ensino } \\
\text { superior }\end{array}$ & $\begin{array}{c}62,5 \%, \text { ensino } \\
\text { fundamental } \\
\text { incompleto, } 59,6 \% \\
\text { completo e } 63,4 \% \\
\text { ensino médio } \\
\end{array}$ & $\begin{array}{l}31,7 \% \text {, ensino } \\
\text { médio }\end{array}$ & $28,6 \%$ \\
\hline $\begin{array}{l}\text { Sampaio e Cruz } \\
\text { (2015) }\end{array}$ & $\begin{array}{c}34,9 \%, 5 \text { a } 8 \text { anos } \\
\text { de estudo }\end{array}$ & $\begin{array}{c}23,1 \%, 5 \text { a } 8 \text { anos } \\
\text { de estudo }\end{array}$ & - & - \\
\hline $\begin{array}{l}\text { Sampaio e Cruz } \\
(2015\end{array}$ & $\begin{array}{c}33,2 \%, 9 \text { a } 12 \\
\text { anos de estudo }\end{array}$ & $\begin{array}{c}42,1 \%, 9 \text { a } 12 \text { anos } \\
\text { de estudo }\end{array}$ & - & - \\
\hline $\begin{array}{l}\text { Miotto, Silotti e } \\
\text { Barcellos (2012). }\end{array}$ & $\begin{array}{c}55,6 \% \text { posto de } \\
\text { saúde }\end{array}$ & $44,4 \%$ & - & - \\
\hline $\begin{array}{l}\text { Sampaio e Cruz } \\
(2015)\end{array}$ & $40,4 \%$ & $59,6 \%$ & - & - \\
\hline $\begin{array}{l}\text { Cardozo et al. } \\
(2016)\end{array}$ & $45,09 \%$ & $54,91 \%$ & - & - \\
\hline Guedes et al. (2012) & $39,5 \%$ & $28,5 \%$ & $\begin{array}{c}46 \% \text { da empresa, } \\
0,4 \% \text { por possui } \\
\text { mais opções de } \\
\text { dentistas, } 1,9 \% \\
\text { convênio do } \\
\text { sindicato da } \\
\text { classe. }\end{array}$ & $\begin{array}{c}\text { 14,2\% em buscam } \\
\text { em hospitais, } \\
10,7 \% \text { com } \\
\text { vizinhos ou } \\
\text { amigos e } 7,1 \% \\
\text { com } \\
\text { farmacêuticos }\end{array}$ \\
\hline $\begin{array}{l}\text { Próprio autor, } \\
\text { (2017) }\end{array}$ & $\begin{array}{c}20,27 \%, \text { renda } \\
\text { entre } \mathrm{R} \$ 3.000,01 \\
-\mathrm{R} \$ 4.000,00\end{array}$ & $\begin{array}{c}\text { 74,36, renda entre } \\
\mathrm{R} \$ 4.000,01-\mathrm{R} \$ \\
8.000,00 \\
\end{array}$ & $\begin{array}{c}18,39 \%, \text { renda } \\
\text { entre R\$ 2.000,01 } \\
-\mathrm{R} \$ 3.000,00 \\
\end{array}$ & $\begin{array}{c}4,55 \%, \text { renda } \\
\text { entre } \mathrm{R} \$ 1.000,01 \\
-\mathrm{R} \$ 2.000,00\end{array}$ \\
\hline $\begin{array}{l}\text { Sampaio e Cruz } \\
\text { (2015) }\end{array}$ & $\begin{array}{c}13,9 \%, \text { renda } \\
\text { familiar entre R\$ } \\
1.501,00 \mathrm{a} \\
2.500,00\end{array}$ & $\begin{array}{c}24,0 \%, \text { renda } \\
\text { familiar entre } \mathrm{R} \$ \\
1.501,00 \text { a } 2.500,00\end{array}$ & - & - \\
\hline
\end{tabular}

Fonte: Organizado pelos autores com base em Guedes et al. (2012); Miotto, Silotti e Barcellos (2012); Sampaio e Cruz (2015); Cardozo et al. (2016) Lima e Oliveira (2017) e os próprios autores (2018).

Nota-se a busca por tratamentos odontológicos categorizando por: 
Grau de instrução: neste estudo 71,54\% com até o ensino médio buscaram no particular. Na pesquisa de Lima e Oliveira (2017) 63,4\% com ensino médio buscaram no particular. Visto por Sampaio e Cruz (2015) com 42,1\% aqueles que possuem de 9 a 12 anos realizaram seus tratamentos odontológicos em consultórios particulares. Percebe-se que os resultados com maiores indicadores apresentam sujeitos com ensino médio buscando particular. Contudo, mencionado anteriormente por Lima e Oliveira (2017), 80,5\% dos motivos da última consulta foram para revisão e prevenção. Sampaio e Cruz (2015) informaram que $10,79,3 \%$ dos sujeitos necessitavam de tratamento dentário.

Público ou particular: Miotto, Silotti e Barcellos (2012) 55,6\% no posto de saúde público. Sampaio e Cruz (2015) 59,6\% no particular. Cardozo et al. (2016) 54,91\% no particular. Conforme apresentado nesse estudo, foi absoluta a procura por tratamentos por meio do particular, independente da renda. Guedes et al. (2012) 39,5\% no público. Nota-se que a busca alterou nas pesquisas apresentadas de 2012 para 2017, do público para o particular.

Renda: Sampaio e Cruz (2015) 24\% com renda familiar entre R\$ 1.501,00 a 2.500,00 e nesse estudo 74,36\% caminhoneiros dos caminhoneiros com renda $R$ \$ 4.000,01 - R\$ $8.000,00$ buscaram tratamentos odontológicos no particular.

Em relação a convênios de saúde odontológicos, Guedes et al. (2012) observou que 40,7\% dos caminhoneiros fazem planos de saúde e odontológico para sua segurança e a de seus familiares e $46 \%$ dos caminhoneiros buscam tratamentos odontológicos por meio do convênio oferecido pela empresa. Nesse presente estudo 18,39\% dos caminhoneiros com renda entre $\mathrm{R} \$ 2.000,01-\mathrm{R} \$ 3.000,00$ e 23,08\% possuindo até o ensino superior buscam atendimento odontológico por meio do convênio. Lima e Oliveira (2017) notou que 31,7\% dos caminhoneiros com ensino médio buscam tratamentos odontológicos por meio do convênio. Levando em consideração as informações observa-se que, os caminhoneiros preocupam-se com seus familiares na obtenção de um plano de saúde e odontológica, e buscam tratamentos nos convênios odontológicos dada as condições: renda entre R $\$ 2.000,01$ - $\mathrm{R} \$ 3.000,0$, benefício do convênio odontológico oferecido pela empresa que trabalha, possuir ensino médio ou superior e família constituída.

Miotto, Silotti e Barcellos (2012) e Monteiro (2013) apresentam em estudo semelhantes sobre condições de saúde bucal dos trabalhadores: 60,43\% dos trabalhadores sentiram dores na boca ou dentes nos últimos 6 (seis) meses, 62,64\% ficaram preocupados ou estressados por problemas bucais ou nos dentes nos últimos 6 (seis) meses, 40,65\% sentiram- 
se enfraquecido fisicamente por não se alimentarem devido dores na boca ou nos dentes nos últimos 6 (seis) meses, 36,26\% irritação ou dificuldade para realizar atividades diárias por dores na boca ou dentes nos últimos 6 (seis) meses, 25,27\% limitação ou incapacidade para realizar atividades diárias por dores na boca nos últimos 6 (seis) meses, $42 \%$ dos sujeitos tiveram dor de dente nos últimos 6 (seis) meses, 23,06\% faltaram no emprego por dor de dente, $10 \%$ afastaram do trabalho por dor de dente e $47 \%$ trabalhadores homens faltam no trabalho por dor ocasionada pelo dente.

Guedes et al. (2012), por meio do estudo com caminhoneiros busca identificar se estes conheciam os serviços prestados pela Atenção à Saúde através dos SUS: 60,8\% conheciam os serviços, 39,2\% não conheciam os serviços, 53,5\% disseram não procurar os serviços, 9,6\% não buscavam as UBS em nenhuma situação, 46,6\% buscavam os serviços, referente a saúde bucal $8,7 \%$ dos caminhoneiros conhecem o serviço e apenas 5,5\% utilizam o serviço e $46 \%$ utilizam o benefício oferecido pela empresa na adesão ao plano de saúde e saúde bucal. Cardozo et al. (2016), em seu estudo com dados da PNS de 2013 percebeu que 45,09\% procurou atendimento de saúde bucal no sistema público e 54,91\% no sistema privado, em 2008 5,2\% da população brasileira apresentou possuir assistência exclusivamente odontológica privada.

\section{CONSIDERAÇÕES FINAIS}

Os objetivos da pesquisa, detectar hábitos na busca de tratamentos de caminhoneiros, foram cumpridos.

Foram feitas análises de fatores que influenciam a busca por tratamentos, como renda e grau de instrução.

As condições de trabalho e a atuação em horários irregulares dificultam o acesso dos caminhoneiros na procura de tratamentos odontológicos nos postos de saúde dentro dos horários estabelecidos para atendimento e locais onde estão instalados, longe das rodovias. Os caminhoneiros consideram também a ausência de estacionamento para caminhões nesses locais. Com isso inviabiliza para maioria dos caminhoneiros a busca por tratamentos odontológicos gratuitos em postos de saúde e SEST SENAT.

Os vínculos trabalhistas dos profissionais caminhoneiros rodoviários de transportes de cargas em 2008, 59,5\% dos caminhoneiros eram autônomos e em 2017, 58,52\% encontramse registrados nas Consolidação das Leis do Trabalho. Com esse novo cenário, possibilitou aos caminhoneiros acesso a convênios odontologicos por meio das empresas. 
Os caminhoneiros buscam tratamentos nos convênios odontológicos, dada às condições: renda entre $\mathrm{R} \$ 2.000,01$ - $\mathrm{R}$ \$ 3.000,00, benefício do convênio odontológico oferecido por meio das empresas que trabalham, possuir ensino médio ou superior e família constituída.

A busca por tratamentos odontológicos no particular está acima dos $50 \%$ entre os caminhoneiros e todas as rendas apresentadas. Observa-se que, os caminhoneiros que possuem ensino médio, nos estudos apresentados, houve um aumento de 2015 a 2017 na busca por tratamento particular.

Com base no estudo sugere-se uma proposta de Políticas Públicas para os caminhoneiros de transporte rodoviário de cargas: a inserção da odontologia do trabalho, isto é, a introdução do cirurgião-dentista na atuação de uma equipe de saúde integral no PCMSO, realizando exames odontológicos admissionais, periódicos e demissionais nos motoristas de caminhões.

\section{REFERÊNCIAS}

CARDOZO, Daniela Peres, SCHAAB, Luana Las, CUNHA, Marina Silva da, COSTA, Cássia Kely Favoretto. Perfil da demanda de saúde bucal no Sistema Único de Saúde (SUS). Revista Orbis Latina v. 6, n. 2 (2016). Disponível em <https://revistas.unila.edu.br/orbis/article/view/597> Acesso em 19 julho de 2017.

CHAVES, Sonia Lima; SANTOS, Carla Lima. Oral health policy in Brazil between 2003 and 2014: scenarios, proposals, actions, and outcomes. Ciência \& Saúde Coletiva, Jun 2017, Vol.22(6). 2017.

GUEDES, Helisamara Mota; PAULA, Alessandra Custódio de; SILVA, Aline Mara da Conceição; ALMEIDA, Martha Elisa Ferreira de. Utilização de serviços de atenção básica à saúde por caminhoneiros. Enfermagem Brasil, v. 11, n. 6, 2012.

GUERRA, Maria Campos; Greco, Rosangela; LEITE, Isabel Gonçalves. Impact of oral health conditions on the quality of life of workers. Ciência \& Saúde Coletiva. Vol.19(12), pp.47774786. 2014.

HOURI, Loliza Chalub Luiz Figueiredo. Tese (doutorado) - Universidade Federal de Minas Gerais. Faculdade de Odontologia. 2015

LIMA, Aline Priscila Dantas; OLIVEIRA, Fabiane Karoline Souza. Autopercepção em saúde bucal de motoristas de caminhão que circulam em Porto Velho-RO. Centro Universitário São Lucas. 2017.

LIMA, Luísa Silva; ARAÚJO, Maristela Santos; CASTRO, Paloma Perez; CANGUSSU, Maria Cristina Teixeira. Condições de saúde bucal de industriários participantes de Programa 
de Saúde Bucal na Empresa. Rev. bras. saúde ocup., São Paulo , v. 40, n. 132, p. 137-

146 ,

Dec.

2015

Disponível

em:

$<$ http://www.scielo.br/scielo.php?script=sci_arttext\&pid=S0303-

76572015000200137\&lng=en\&nrm=iso>. Acesso em: 31 de outubro de 2017.

MIOTTO, Maria Helena Monteiro de Barros; SILOTTI, Jean Carlos Bazoni; BARCELLOS, Ludmilla Awad. Dor dentária como motivo de absenteísmo em uma população de trabalhadores. Ciênc. saúde coletiva, Rio de Janeiro, v. 17, n. 5, p. 1357-1363, May 2012 - Disponível em: $\quad$ http://www.scielo.br/scielo.php?script=sci_arttext\&pid=S1413$\underline{81232012000500029 \& \operatorname{lng}=e n \& n r m=i s o>}$. Acesso em 19 de maio de 2017.

MOTA, Júnia Nara Gonçalves; WANDERLEY, Flávia Godinho Costa; SILVA, Ricardo Araújo da; ALMEIDA, Tatiana Frederico de. Absenteísmo por causa odontológica: uma revisão de literatura relacionada à ausência no trabalho e à saúde bucal do trabalhador. RFO UPF, Passo Fundo, v. 20, n. 2, ago. 2015 . Disponível em: $<$ http://revodonto.bvsalud.org/scielo.php?script=sci_arttext\&pid=S141340122015000200022\&lng=pt\&nrm=iso> . Acesso em: 19 de maio 2017.

PENTEADO, Regina Zanella; GONÇALVES, Claudia Giglio de Oliveira; COSTA, Daniele Damaris da; MARQUES, Jair Mendes . Trabalho e saúde em motoristas de caminhão no interior de São Paulo. Saude soc., São Paulo, v. 17, n. 4, p. 35-45, Dec. 2008 Disponível em: $<$ http://www.scielo.br/scielo.php?script=sci arttext\&pid=S0104$12902008000400005 \& \operatorname{lng}=$ en\&nrm=iso $>$. Acesso em 10 de abril de 2017.

SAMPAIO, Danielle Maria Leite e CRUZ, Lusanira Maria da Fonsêca de Santa. Perfil de utilização dos serviços odontológicos públicos e privados pela população adulta brasileira. Revista Brasileira de Pesquisa em Saúde/Brazilian Journal of Health Research, v. 16, n. 3, 2015.2 Disponível em: <http://www.periodicos.ufes.br/RBPS/article/download/10146/6888> Acesso em 19 de Julho de 2017

SOBRINHO, José E. L. Das Bocas Famintas às Sorridentes: Uma Análise Da Política Nacional de Saúde Bucal do Brasil. 2014. Tese (Doutorado). Fundação Oswaldo Cruz Centro de Pesquisas Aggeu Magalhães Doutorado em Saúde Pública. Disponível em: <http://www.cpqam.fiocruz.br/bibpdf/2014lorena-sobrinho-je.pdf $>$. Acesso: em 23 de abril de 2017.

SANTOS, Nellis Oliveira; KAWAMOTO, Luiz Teruo; BONINI, SOUGUELLIS, Hewely Cardoso; BONINI, Luci Mendes de Melo. Condições de Saúde e Trabalho dos Caminhoneiros No Brasil. In: III CONGRESSO INTERNACIONAL DE LOGÍSTICA E OPERAÇÕES DO IFSP DE SUZANO, 3, 2017, Suzano. Resumos... Instituto Federal de Educação, Ciência e Tecnologia de São Paulo, 2017.

VENDRAME, Édina. Efeitos da posição social da infância e da vida adulta na perda dentária, nas doenças crônicas e na qualidade de vida relacionada a saúde bucal. 2016. Dissertação (Mestrado). Faculdade e Odontologia Programa de Pós-Graduação em Odontologia. Disponível em: <http://www.lume.ufrgs.br/handle/10183/151438>. Acesso em 10 de abril de 2017 
VIEIRA, Vladen; ANDRADE, Flávia Reis de; CASTRO, Cláudio Gastão Junqueira de; BIGHETTI, Tania Izabel; NARVAI, Paulo Capel. Municipalização de serviços de saúde segundo profissionais de saúde bucal em um município do interior do estado de São Paulo, Brasil. Saúde e Sociedade. Vol.22(3), pp.795-803. 2013. 